\title{
Leather for motorcyclist garments: Multi-test based material model fitting in terms of Ogden parameters
}

\author{
T. Bońkowski ${ }^{a, *}$, L. Šoltés ${ }^{a}$, L. Hynčík ${ }^{a}$, R. Kottner ${ }^{a}$, P. Kochováa ${ }^{a}$

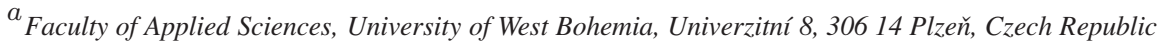

Received 31 July 2017; accepted 4 December 2017

\begin{abstract}
This paper is focused on experimental testing and modeling of genuine leather used for a motorcycle personal protective equipment. Simulations of powered two wheelers (PTW) accidents are usually performed using human body models (HBM) for the injury assessment equipped only with the helmet model. However, the kinematics of the PTW rider during a real accident is disturbed by the stiffness of his suit, which is normally not taken into account during the reconstruction or simulation of the accident scenario. The material model proposed in this paper can be used in numerical simulations of crash scenarios that include the effect of motorcyclist rider garment. The fitting procedure was conducted on 2 sets of samples: 5 uniaxial samples and 5 biaxial samples. The experimental characteristics were used to obtain the set of 25 constitutive material models in terms of Ogden parameters.
\end{abstract}

(c) 2017 University of West Bohemia. All rights reserved.

Keywords: genuine leather, tensile test, Ogden model, hyperelastic materials

\section{Introduction}

At the turn of 2015/2016, the number of motorcycle accidents has decreased in the Czech Republic by $16 \%$. The $17 \%$ of all road fatalities in the EU (2016) occur with some contribution of the powered two wheelers (PTW). Most of them (55\%) happened on rural roads [13]. The products made from leather are very often used in the motorcycle sport, however, many motorcycle riders consider the motorcycle protective clothing only as a protection against the bad weather conditions [10]. The manufacturers and developers of motorcyclist outfits often follow only the standardized test procedures $[12,14]$. For motorcycle riders, both the safety and the comfort are very important. This paper is focused on the experimental measurement and fitting material model of leather. The experimental part presents two methods: standardized uniaxial test and non-standardized biaxial test. The experimental results were used to fit the Ogden parameters of materials models. The correlation between experiments and models were compared by the finite element method (FEM) simulations.

The leather is manufactured from the skin of animals. The skin is the biggest organ of all multicellular animals. The basic function of the skin is to protect the body against the mechanical damage, often is called a body armor. The skin has three basic layers: epidermis, dermis, hypodermis (people), subcutaneous layer (animals). The essential constituent of leather is tanned-collagen fiber-network (around $99 \%$ by weight), with $300 \mathrm{~nm}$ in length and $1.5 \mathrm{~nm}$ in diameter $[1,5]$. The collagen can be characterized as a viscoelastic material with hysteresis and stress relaxation [6]. Despite the collagen, low percentage of the elastin can be found in the leather structure as well [11]. There are three sub-processes which transform the animal skin into the

\footnotetext{
*Corresponding author. Tel.: +420 377639 181, e-mail: tomasz@ntis.zcu.cz. https://doi.org/10.24132/acm.2017.390
} 


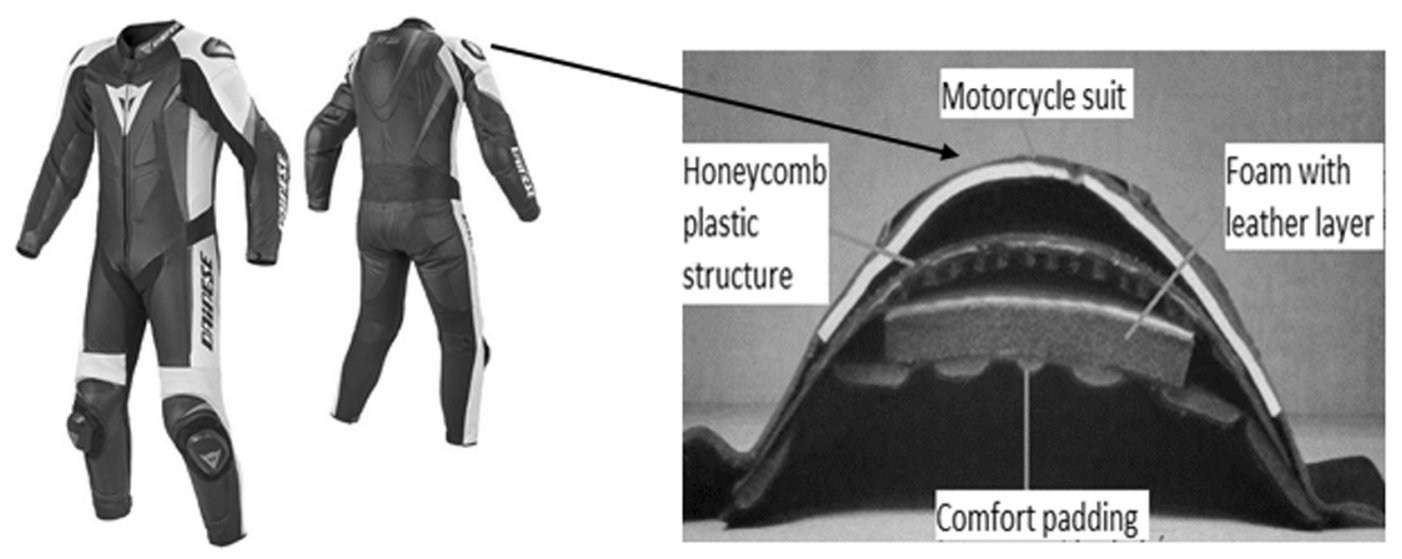

Fig. 1. The leather in a motorcycle garment [15]

leather: preparatory stages, tanning, and crusting. Mechanical properties of the leather as strain rate and strength are dependent on the direction of the leather cut, humidity, age and health of the animal, thus, the leather is not a uniform material from a structural perspective [7]. Actually, the mechanical properties in the tension of the leather are tested according to the standard ISO 3376. This test is based on the one uniaxial testing and concerns only the ultimate strength. Samples in the form of bones are fixed in clamps of the testing machines [14]. However, it is proved that mechanical properties of the leather in two perpendicular cuts are very different [3]. Fig. 1 shows the possibility of using the leather layer as a part of the motorcycle protector. In this case, it prevents foam penetration by the honeycomb structure.

\section{Method}

The fitting procedure of the Ogden material parameters was conducted under the Virtual Performance Solution package (VPS). According to the software manual [16], the Ogden material is described by material type 37 - solid hyperelastic constitutive material model. Parameters, which describes the constitutive relationship ( $\mu$ - Ogden parameter, $\alpha$-Ogden exponent, $N-$ model order), could be set explicitly, or identified by built-in optimization algorithms from prescribed materials tests. Uniaxial and biaxial tests were used in this work. Before the tests samples were conditioned for 48 hours in the standard atmosphere $\left(23{ }^{\circ} \mathrm{C} / 50 \%\right.$ relative humidity). The tests were performed under identical conditions.

\subsection{Uniaxial tensile test}

The uniaxial test of the leather samples was made in line with the procedure described in the ISO standard [14]. Tested samples were bone-shaped with standardized "large" dimensions. The "large" dimensions were selected due to initial slippage of the test piece in the jaws [14]. This testing procedure is an extension of earlier author's work [2,3]. Tests were made on the Zwick/Roell Z050 testing machine equipped with the mechanical extensometer. During the tests, the jaw separation speed was fixed to $100 \mathrm{~mm} / \mathrm{min}$. To achieve the statistical significance, 5 samples were used for this procedure. The selection of the samples is not in line with the ISO standard ( 3 samples parallel to the backbone and 3 perpendicular), due to the lack of access to the entire animal hide. This deviation did not have an influence on the main aim of the work (the motorcycle suits are made from leather pieces cut in the way which utilize the hide to maximum). These samples were stretched to ultimate damage. This procedure allowed to 


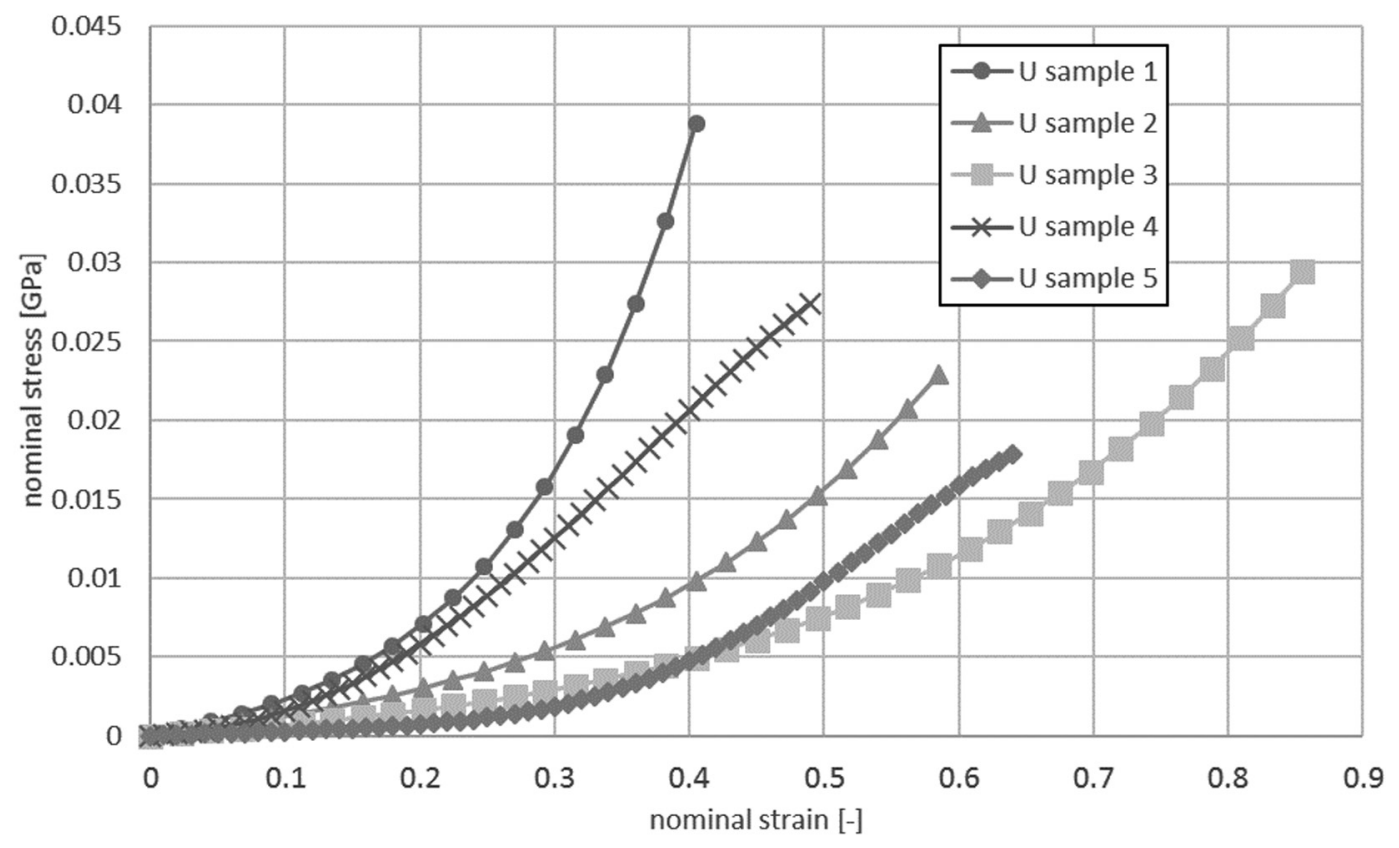

Fig. 2. Extension characteristics of uniaxial samples (U sample)

obtain force-extension characteristics in the maximum possible range of extension. By dividing the force by the initial cross-section area and the extension by initial distance between the extensometer clamps, the nominal strain-nominal stress relationship was obtained (Fig. 2). According to the [3] this wide range of the results (Fig. 2) covers $68 \%$ of the results distribution in stiffness.

\subsection{Biaxial tensile test}

The biaxial tensile test was performed on cross-shaped randomly cut samples [3]. Due to architecture restrictions of the Zwick/Roell Z050, this machine could not be used to biaxial tests (lack of the second testing axis). Thus the 574LE2 Biomechanics and Tissue Tester from TESTRESOURCES Company was used. The separation speed of each pair of jaws was set to $100 \mathrm{~mm} / \mathrm{min}$. The machine was equipped with the 4 independent force cells (one for each jaw) and video extensometer. To obtain the nominal stress time series from above-mentioned signals, following expression was used:

$$
\sigma_{\text {nom }}(t)=\frac{1}{4} \sum_{N=1}^{4} \frac{F_{N}(t)}{A_{N}^{0}},
$$

where $F_{N}(t)$ is the force recorded from one of the 4 force-cells and $A_{N}^{0}$ is the initial cross-section area of the sample limb connected to the $N$-th force-cell.

The nominal strain time series was calculated by the expression:

$$
\varepsilon_{n o m}(t)=\frac{1}{2} \sum_{M=1}^{2} \frac{\Delta l_{M}}{L_{M}^{0}},
$$

where $\Delta l_{M}$ is a change in the sample length on the $M$-th axis and $L_{M}^{0}$ is the initial length of the sample in the $M$-th axis. 


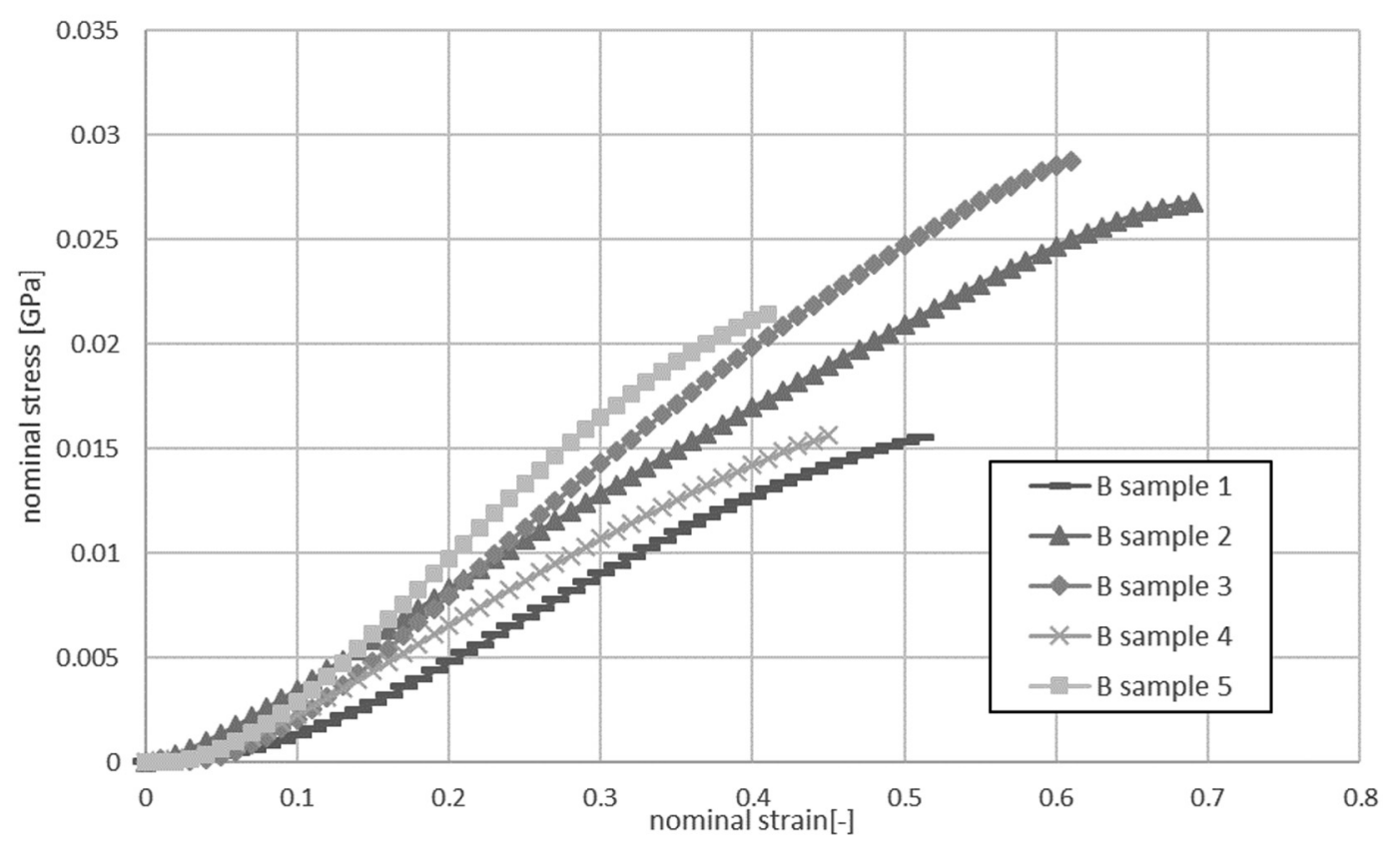

Fig. 3. Extension characteristics of biaxial samples

For each time step of the experiment, the expressions (1), (2) were calculated and correlated in time (Fig. 3). This procedure was applied to 5 biaxial samples (B sample).

\subsection{The hyperelastic Ogden material model}

The material type 37 implemented in the VPS package is Ogden-based hyperelastic solid which is using following the formulation to describe strain energy functional [9]:

$$
W=\sum_{A=1}^{3} \sum_{i=1}^{N} 2 \frac{\mu_{i}}{\alpha_{i}}\left(\overline{\lambda_{A}^{\alpha_{i}}}-1\right)+\frac{K}{2}(J-1)^{2},
$$

where $\bar{\lambda}_{A}=J^{-1 / 3} \lambda_{A}, J=\operatorname{det} F, \bar{C}=\bar{F}^{T} \bar{F}, \bar{F}=J^{-1 / 3} F, \lambda_{A}$ are eigenvalues of the deformation gradient matrix $F, \mu_{i}$ is the Ogden parameter, $\alpha_{i}$ is the Ogden exponent, $N$ is the model order and $K$ is the bulk modulus.

\subsection{Model parameters fitting}

The fitting procedure was done under the VPS pre-processor, which provides a wide range of optimization algorithms. For the multi-test based material fitting, the linear least-square method occurs not to be enough. The solution of the nonlinear least-square problem is not trivial, for this problem 3 main methods were chosen: the Levenberg-Marquardt method (damped GaussNewton algorithm) [4], the Hybrid Method (combination of the Levenberg-Marquardt method and Quasi-Newton method) and the Separable Nonlinear Least Square Method [8, 16].

Fig. 4 shows the comparison of the fitting error (the L2 norm) between the different numerical methods and the Ogden models with the different prescribed order. There is no significant difference of error between the Ogden models in the same order obtained by different nonlinear least-square problem solution methods. The raising of the order 2 did not provide any decrease in error. The model of order one looks to have the error two-times higher, but only this model provides the stability in the wide range of strains $(-0.9 \div 0.9)$. 


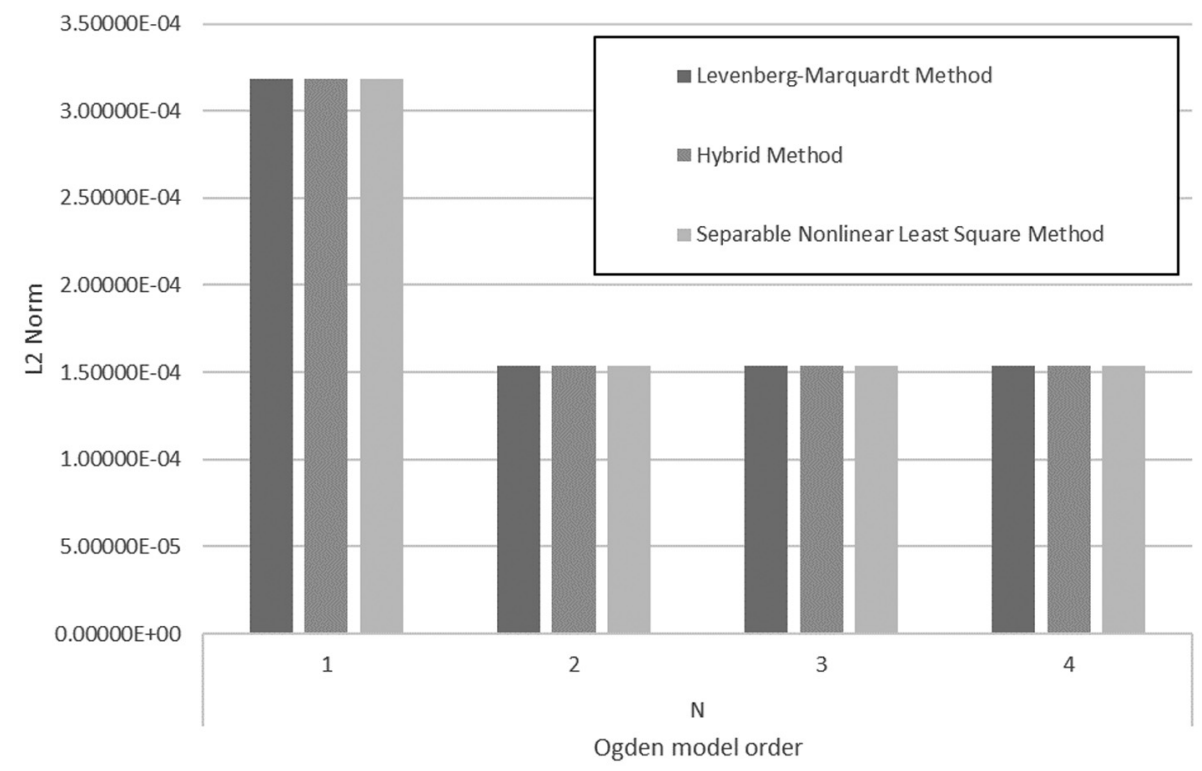

Fig. 4. The L2 norm sensitivity analysis for the Ogden model order and solution method

\subsection{Finite element method simulation}

To validate the fitting procedure, the finite element simulations were performed. The behaviors under the uniaxial and biaxial loading were tested. These tests were conducted on one 8-node element with linear integration.

\subsubsection{One-element uniaxial simulation}

The one-element uniaxial material calibration was done on the single solid 8-node element. The dimensions of the element were $10 \times 10 \times 1 \mathrm{~mm}$. The nodes were fixed according to Fig. 5 . The constant speed displacement was applied to 4 nodes on the $10 \times 1 \mathrm{~mm}$ element wall. The speed was the same as in the experimental tests $(100 \mathrm{~mm} / \mathrm{min})$. The simulation was conducted up to 6 seconds.

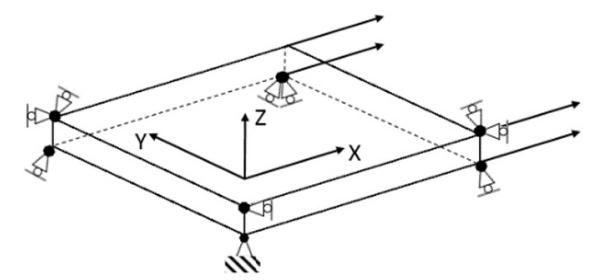

Fig. 5. One-element uniaxial tension simulation setup

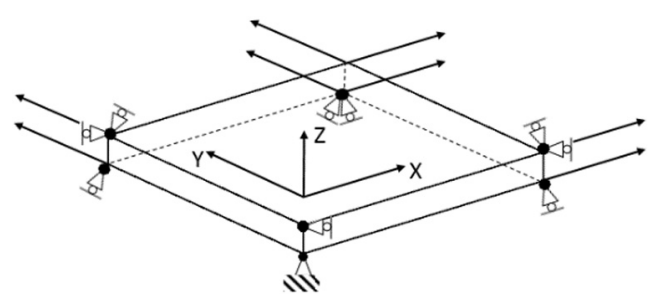

Fig. 6. One-element biaxial tension simulation setup

\subsubsection{One-element biaxial simulation}

The calibration under the biaxial loading was made on the same element like in the uniaxial case. The only difference was in the loading conditions. The biaxial loading conditions are depicted by the black arrows in Fig. 6 . 


\section{Results}

\subsection{Ogden parameters fitting}

The fitting results are 25 Ogden constitutive material models of motorcycle garment leather (Table 1). Each model was based on two experimental curves (one biaxial and one uniaxial). Due to instability in high strains (by means of the Drucker stability), the rank equal to one was chosen for the models.

Table 1. Considered 25 cross-test Ogden material models for the leather (Us - uniaxial sample, Bs-biaxial sample)

\begin{tabular}{|c|c|c|c|c|c|c|}
\hline $\begin{array}{c}\text { Sample } \\
\text { number }\end{array}$ & Us1 & Us2 & Us3 & Us4 & Us5 & $\begin{array}{c}\text { Ogden } \\
\text { parameter }\end{array}$ \\
\hline \multirow{2}{*}{ Bs1 } & $1.35 \mathrm{e}-03$ & $9.41 \mathrm{e}-04$ & $1.50 \mathrm{e}-03$ & $1.05 \mathrm{e}-03$ & $9.99 \mathrm{e}-04$ & $\mu[\mathrm{GPa}]$ \\
\cline { 2 - 7 } & $6.01 \mathrm{e}+00$ & $6.35 \mathrm{e}+00$ & $4.48 \mathrm{e}+00$ & $6.94 \mathrm{e}+00$ & $5.41 \mathrm{e}+00$ & $\alpha[-]$ \\
\hline \multirow{2}{*}{ Bs2 } & $3.34 \mathrm{e}-03$ & $2.54 \mathrm{e}-03$ & $5.34 \mathrm{e}-03$ & $3.04 \mathrm{e}-03$ & $-9.67 \mathrm{e}-03$ & $\mu[\mathrm{GPa}]$ \\
\cline { 2 - 7 } & $3.87 \mathrm{e}+00$ & $4.29 \mathrm{e}+00$ & $2.43 \mathrm{e}+00$ & $4.13 \mathrm{e}+00$ & $-1.02 \mathrm{e}+00$ & $\alpha[-]$ \\
\hline \multirow{2}{*}{$\mathbf{B s 3}$} & $2.65 \mathrm{e}-03$ & $2.22 \mathrm{e}-03$ & $-4.53 \mathrm{e}-02$ & $2.26 \mathrm{e}-03$ & $-6.70 \mathrm{e}-03$ & $\mu[\mathrm{GPa}]$ \\
\cline { 2 - 7 } & $4.85 \mathrm{e}+00$ & $4.92 \mathrm{e}+00$ & $-2.93 \mathrm{e}-01$ & $5.23 \mathrm{e}+00$ & $-1.45 \mathrm{e}+00$ & $\alpha[-]$ \\
\hline \multirow{2}{*}{$\mathbf{B s 4}$} & $1.14 \mathrm{e}-03$ & $1.50 \mathrm{e}-03$ & $2.04 \mathrm{e}-03$ & $9.47 \mathrm{e}-04$ & $2.00 \mathrm{e}-03$ & $\mu[\mathrm{GPa}]$ \\
\cline { 2 - 7 } & $7.23 \mathrm{e}+00$ & $5.32 \mathrm{e}+00$ & $3.90 \mathrm{e}+00$ & $7.68 \mathrm{e}+00$ & $3.78 \mathrm{e}+00$ & $\alpha[-]$ \\
\hline \multirow{2}{*}{$\mathbf{B s 5}$} & $1.15 \mathrm{e}-03$ & $7.10 \mathrm{e}-03$ & $7.72 \mathrm{e}-03$ & $1.70 \mathrm{e}-03$ & $-8.09 \mathrm{e}-03$ & $\mu[\mathrm{GPa}]$ \\
\cline { 2 - 7 } & $8.36 \mathrm{e}+00$ & $1.98 \mathrm{e}+00$ & $1.70 \mathrm{e}+00$ & $6.44 \mathrm{e}+00$ & $-1.31 \mathrm{e}+00$ & $\alpha[-]$ \\
\hline
\end{tabular}

The instantaneous shear modulus for the Ogden hyperelastic material can be defined as follows:

$$
G_{0}=\sum_{i=1}^{N} \mu_{i} \alpha_{i} .
$$

Fig. 7 presents the distribution of the instantaneous shear modulus of the identified models of the leather in terms of Ogden parameters. The average of the instantaneous shear modulus of identified models was found on value $9.9 \mathrm{MPa}$. The model based on the uniaxial sample 5 and the biaxial sample 2 appeared to be on the average of the distribution. This model was chosen as the mean model of the motorcycle leather.

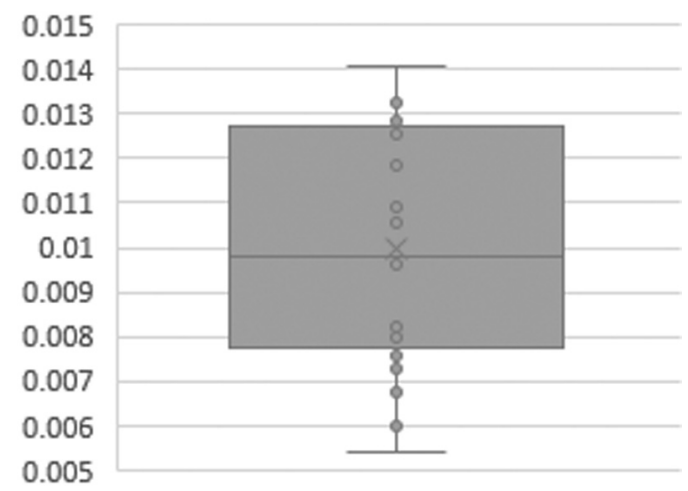

Fig. 7. Box plot of models instantaneous shear modulus [GPa] 


\subsection{FEM simulation results}

FEM simulations were conducted according to the adopted method. The Ogden material was applied to an 8-node solid hexahedron element.

The response of the model under the biaxial loading is acceptable up to the strain of 0.7. The model did not represent the uniaxial response in the wide range of strains. Fig. 8 shows that the identified model estimates well the response of the real sample for uniaxial loading only up to strain of 0.45 .

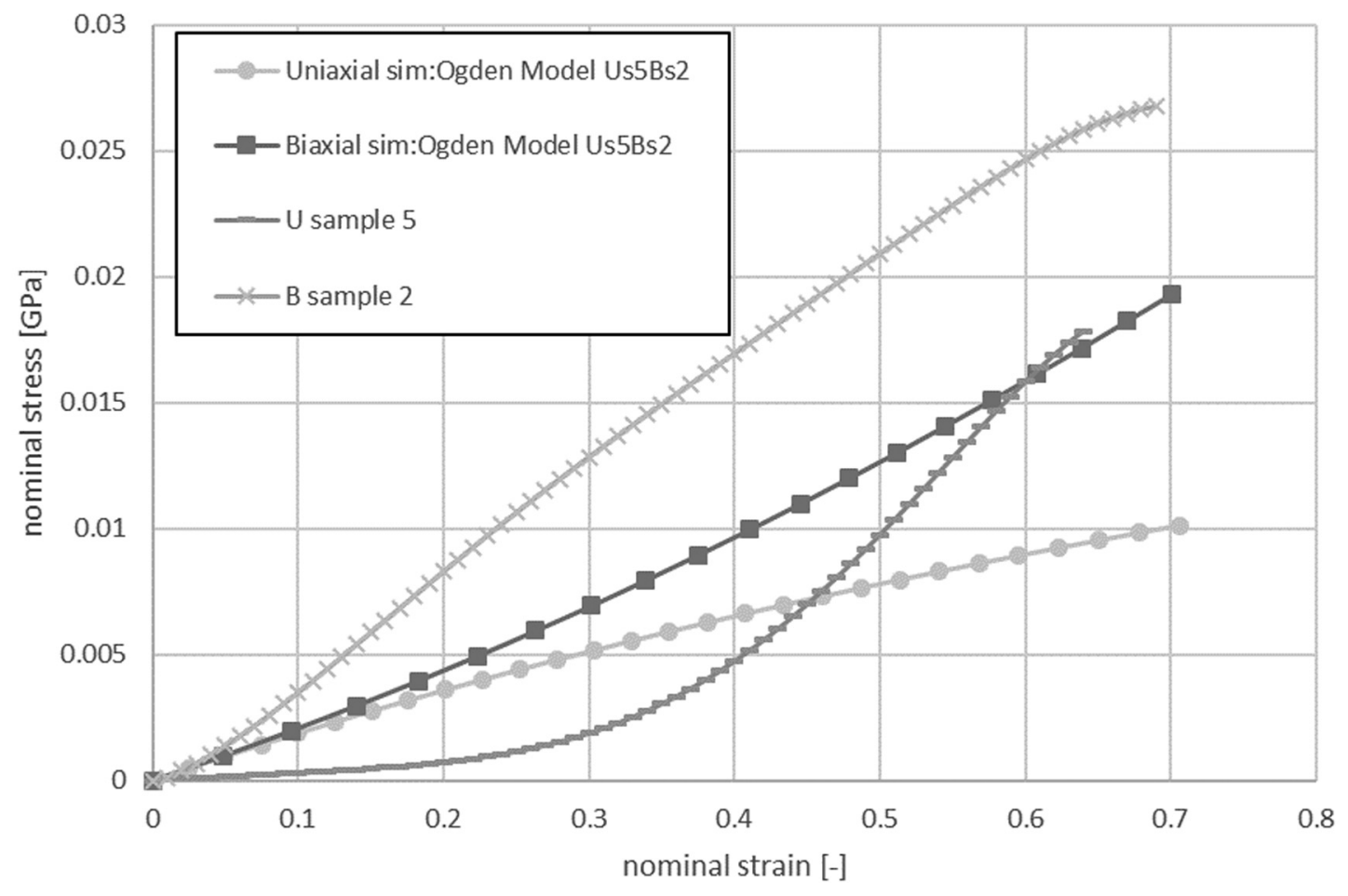

Fig. 8. One-element simulations results

\section{Conclusions}

The work shows the procedure of multi-test constitutive material model fitting in terms of the Ogden parameters. This method was applied for obtaining a numerical model of leather material.

This material can be used in future for the modeling of protective jackets and suits. The numerical protective garments could be coupled with human body models (HBM) to improve the realistic behavior of the HBM during the powered two wheelers (PTW) crash simulations.

\section{Acknowledgements}

The work has been supported by the research project LTC17001 "Exploitation of virtual human model for reducing injury risk of PTW riders" as the national link to the COST Action TU1407 "Scientific and technical innovations for safer Powered Two Wheelers (PTW)" (T. Bońkowski, L. Hynč́k, R. Kottner), the internal grant project SGS-2016-059 "Computer modelling and monitoring of human body used for medicine" (T. Bońkowski, L. Šoltés) and by the project LO1506 of the Czech Ministry of Education, Youth and Sports under the program NPU I. (P. Kochová). 


\section{References}

[1] Alberts, B., Bray, D., Hopkin, K., Johnson, A., Lewis, J., Raff, M., Roberts, K., Walter, P., Essential cell biology, Espero Publishing, 2005.

[2] Bońkowski, T., Kottner, R., Krystek, J., Hubík, L., Görner, T., Hynčík, L., Tensile test of motorcycle garment leather, Proceedings of the Conference on Experimental Stress Analysis (EAN 2016), Srni, 2016.

[3] Bońkowski, T., Šoltés, L., Kochová, P., Kottner, R., Hynčík, L., Motorcycle suits: Uniaxial and biaxial tensile tests of leather, Proceedings of the Conference on Experimental Stress Analysis (EAN 2017), Novy Smokovec, 2017.

[4] Levenberg, K., A method for the solution of certain problems in least-squares, Quarterly of Applied Mathematics 2 (1944) 164-168. https://doi.org/10.1090/qam/10666

[5] Li, Z., Paudecerf, D., Yang, J., Mechanical behaviour of natural cow leather in tension, Acta Mechanica Solida Sinica 22 (1) (2009) 37-44. https://doi.org/10.1016/S0894-9166(09)60088-4

[6] Liu, C., Lantona, N., DiMaio, G., Cook, P., Viscoelasticity studies for a fibrous collagen material: Chrome-free leather, Journal of Materials Science 42 (20) (2007) 8509-8516. https://doi.org/10.1007/s10853-007-1744-1

[7] Mutlu, M., Ork, N., Yegin, O., Basa, S., Mapping the variations of tensile strength over the area of sheepskin leather, Annals of the University of Oradea Fascicles of Textiles, Leatherwork, 2014.

[8] Nielsen, H. B., Separable nonlinear least-squares, IMM, DTU Report IMM-REP-2000-01, 2000.

[9] Ogden, R. W., Non-linear elastic deformations, New York, Dover Publications Inc., 1984.

[10] Rome, L., Ivers, R., Fitzharris, M., Du, W., Haworth, N., Heritier, S., Motorcycle protective clothing: Protection from injury or just the weather?, Accident Analysis and Prevention 43 (6) (2011) 1983-1990.

[11] Standring, S., et al., Gary's Anatomy, Elsevier, 2005.

[12] ASTM D7255-14, Standard test method for abrasion resistance of leather (Rotary Platform, Abraser Method), ASTM International, West Conshohocken, PA, 2014.

[13] 2016 road safety statistics, retrieved July 26, 2017, from http://europa.eu/rapid/press-release_MEMO-17-675_en.htm.

[14] Leather - Physical and mechanical tests - Determination of tensile strength and percentage extension, ISO 3376:2002(E).

[15] Motorcycle clothing, retrieved July 31, 2017, from https://www.dainese.com.

[16] Virtual Performance Solution 2014 - Solver reference manual, Volume III, 2014. 